\title{
Research on Influence of Different Damping Models on Anti-seismic Structures
}

\author{
ChangXian Zhou ${ }^{\mathrm{a}}$, ShaoPeng Zheng ${ }^{2, \mathrm{~b}}$ and CongRongRen ${ }^{3, \mathrm{c}}$ \\ Xiamen city Jimei District Sheng Road No. 669 Xiamen Research Center of Seismology Survey \\ a41500619@qq.com, ${ }^{\mathrm{b}} 41500619 @ q q . c o m,{ }^{c} 41500619 @ q q . c o m$
}

Keywords: Damping Model, Elastic-plastic Analysis, Plane Frame Structure

\begin{abstract}
The analysis and design methods based on energy not only is clear in concept and simple in form, but also better reflects the intensity of ground motion, spectral characteristics, in particular structural cumulative damage failure caused by the duration of strong earthquake. Therefore, seismic response analysis based on energy has been increasingly paid concern by domestic and international earthquake engineering research community, and some progress of this field has been made. Considering changes of ground motion factors changing law of total input energy and its distribution and structural dynamic properties, of plane frame structure for different damping model is analyzed, and then it is obtained that the difference using of the total input energy and its distribution as a result of different damping model selection, reaching a preliminary understanding of these quantitative and qualitative differences in the law. A new method was proposed to compute the damping coefficients in linear dynamic analysis. The method is verified by comparing to the mode-superposition approach.
\end{abstract}

\section{Theoretical Introduction of Damping Mechanism and Model}

Vibration damping system is a reflection of the structure of the process parameters of the energy dissipation characteristics, is an important factor affecting the structural vibration response and the seismic response. There exists a sufficient damping, mean that a structure capable of absorbing more in the vibration energy can be effectively reduced and the stress amplitude. In general, in the vibration analysis of the structure, as compared with the elastic force and the inertial force, the damping force is smaller in magnitude. However, under certain conditions, the damping factor will play a very important role. If no damping force exists, when the resonance vibration system to reach a very large amplitude. Also damping vibration of the structure can be improved, and energy for an earthquake, collision has damaging effects on the structure of the vibration damping can be utilized to absorb consumption.

Seismic structure is essentially the process of passing an energy transformation and consumption, therefore, in recent years on energy analysis method in seismic design of domestic and international issues of common concern by the earthquake engineering, is considered the future development direction of the structural design theory. Impact energy damping is a structure, the mechanism through additional impact block, the vibrational energy of the primary system to convert the vibration energy impact block, so as to achieve the purpose of reducing the vibration of the main system. Project impact damper can be set to get the shock damping, for example, sand, fine gravel, lead balls or other piece of metals, and even carbide can be used as an impact block ${ }^{[1]}$.

Damping theory is not based on what kind of damping phenomenon could inclusive and interpretation. More importantly, the main purpose of the seismic response analysis is to obtain the reaction of people are concerned about the amount of the structure, rather than the interpretation of the damping process, so when selecting damping models are often more focused on convenient mathematical treatment, and then in accordance with the principle of a structural response to determine which indicators equivalent damping parameters.

\section{Finite Element Analysis}

ABAQUS is the powerful engineering simulation software based on the finite element method, 
which can solve relatively simple linear analysis of nonlinear analog to a variety of problems, such as challenging. ABAQUS offers concrete constitutive model has smeared cracking model for concrete, concrete cracking model and elastic-plastic damage constitutive model. Smeared cracking model is suitable for low confining concrete under monotonic loading. However, this paper is nonlinear dynamic time history of reinforced concrete structure analysis, so the use of elastic-plastic damage constitutive model is more applicable ${ }^{[2]}$.

Calculate the seismic response of frame structure, which normally takes under different circumstances, such as structures, structural features, the computer model of choice for computing capacity, commonly used calculation model are: inter laminar shear model truss model, single-column frame models. Inter laminar shear layer model as the basic unit, the focus on quality floor houses a floor and roof height that beam stiffness is infinite, no bending, corresponding unit only involves an interlayer shear and drift. In the seismic response of structures using inter laminar shear model can greatly reduce the number of degrees of freedom force structure, improve computational efficiency. Truss rod model as the basic unit structure, beams, columns, walls are simplified into a one-dimensional poles expressed its axis, which is the quality of the heap at the nodes or the use of poly consider the unit mass matrix rod mass distribution. Plane frame model closer to the actual structure, the result could reflect changes in the structure of the whole process and each rod in the seismic action.

Finite element analysis model to calculate the energy of the past, it is often the beams as a unit, and the quality of the beams are focused to both ends of the beams, using a lumped mass matrix and cohesion algorithms programmed to calculate the stiffness matrix of energy, Such calculations may lead to large errors. This article will be beamed ABAQUS finite element subdivision unit, you can observe the development of its plasticity, and consistent mass matrix and stiffness matrix is consistent calculation. ABAQUS finite element modeling software, but not the structure of the material departure from the constitutive relation to section beams treated as a unit of the restoring force model, the quality of the beams to the ends of beams of concentrated, Applied Materials recovery force model, the beams for unit subdivision, consistent mass matrix and stiffness matrix, because too many assumptions to avoid errors caused by the increase ${ }^{[3]}$. Fig. 1 shows the element numbering of structure.

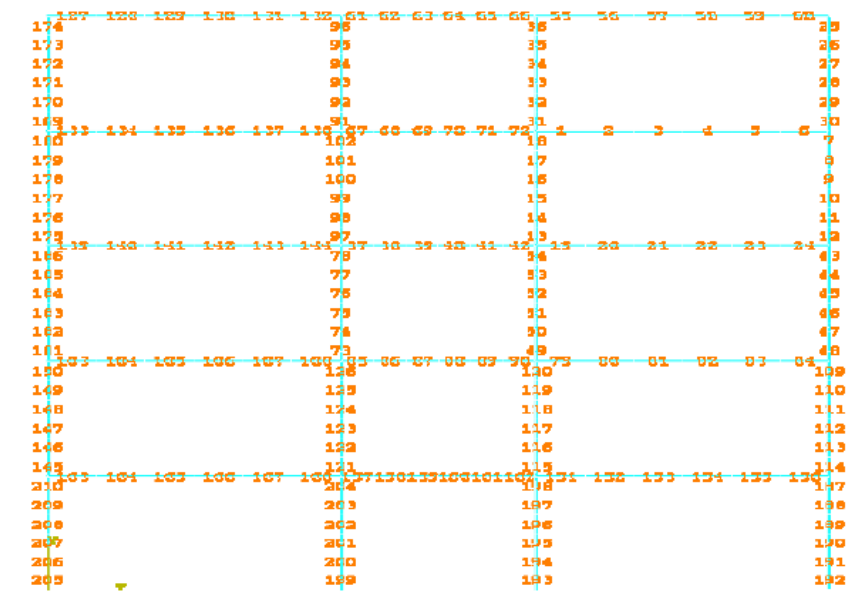

Fig. 1.The Element numbering of Structure

\section{The Seismic Response Analysis of Energy Structure}

After entering the plastic structure, kinetic and elastic strain energy decrease in the proportion of the total energy in the energy structure of the seismic input to rely mainly on the inelastic deformation and damping consumption structure. Solving multi-degree of freedom structure of each energy term is more complex, how to calculate the actual construction process seismic response of each energy term is related to the implementation of energy-based seismic design method of key issues. Scholars agree that the main characteristics of ground motions can land vibration amplitude spectrum and duration of three basic elements to represent ${ }^{[4]}$. 
Peak acceleration was first proposed, is the most intuitive given the magnitude of ground motion. With in-depth study of ground motion, based on different research purposes and definition of the magnitude proposed a variety of angles, so that the definition of the magnitude of the expansion from a single generation, such as the maximum value to some sense. When the earthquake who generally refers to the duration of strong earthquakes, the impact on the structure mainly in the non-linear reaction stage structure. Even in the structure does not reach the maximum deformation under static reaction, whereas the energy dissipated due to plastic deformation up to a certain limit, local structure also occur even overall failure, collapse. Duration of a wide range of definitions, generally divided into time and reaction time record holders hold. When the record is held by the acceleration of ground motion records obtained by direct treatment, only concerned itself with the ground motion recorded; the reaction is a record holder as the input, the reaction to the amount specified in the structure, and it is not only ground motion, but also on the structure of matter and motion characteristics related.

Earthquake ground motion is a wide band of non-stationary random vibration, affected by many factors, such as the location of earthquake faults, plate movement form, distance, wave pathway geological conditions, soil structure and categories and other venues. Often with earthquake strong motion records obtained at the same site is not the same, and therefore can't accurately predict the future of ground motion for each construction site. Studies have shown that, although the future of the construction site of ground motion is difficult to accurately determine the quantitative, but as long as the correct choice of the main parameters of ground motion, and the choice of the seismic wavelet this meet these key parameters, the time history analysis results can be truthfully reflected in future earthquakes Under the effect of the structural response, precision engineering to meet the need.

\section{Seismic Response Calculation of the Structure}

After the structure to absorb seismic energy input to it, only a small portion is converted into kinetic energy and elastic strain energy, mostly dissipated by damping and plastic deformation. Importance of damping and damping energy is well known, but due to the complexity of the damping mechanism, even if the elastic stage, damping term is difficult to determine. Although the damping itself is an objective reality, but because of its very complex mechanism, not like other dynamic characteristics of the structure of mass, stiffness, etc. can be calculated by comparing the same exact way, and therefore the damping in engineering practice often abstract for some convenient The mathematical model, based on the principle of reaction with equivalent physical quantity to determine the structure parameter. Not only the physical characteristics of the damper and the structure itself, but also with the target and analysis methods to determine the equivalent damping when relevant ${ }^{[5]}$.

Damping energy and hysteretic energy increases monotonically with time t, the maximum achieved at the end of the earthquake; elastic strain energy t fluctuate over time under the zero line hysteretic energy can effectively reflect the accumulated damage plastic, is the most projects. Energy response indicators meaning can affect the response duration of strong earthquakes on the structure. After entering the plastic structure, kinetic and elastic strain energy decrease in the proportion of the total energy in the energy structure of the seismic input to rely mainly on the inelastic deformation and damping consumption structure. Solving multi-degree of freedom structure of each energy term is more complex, how to calculate the actual construction process seismic response of each energy term is related to the implementation of energy-based seismic design method of key issues. Generally in the appropriate simplifying assumptions, the combined power differential equations to solve.

In order to understand the failure mechanism and the use of multi-degree of freedom system based seismic design method of energy system to a multi-degree of freedom structural design, analysis model for the selection of different damping effect on the components of the energy distribution structure is based on an accurate understanding of further multi-degree of freedom system of accumulated damage energy (hysteretic energy) distribution between the structural 
members of the law. Based important factors in the structural damping energy analysis, analysis of the impact of differences in model selection damping energy distribution of the structural members is also very important. Fig.2 shows the proportion of beam and column energy dissipation.

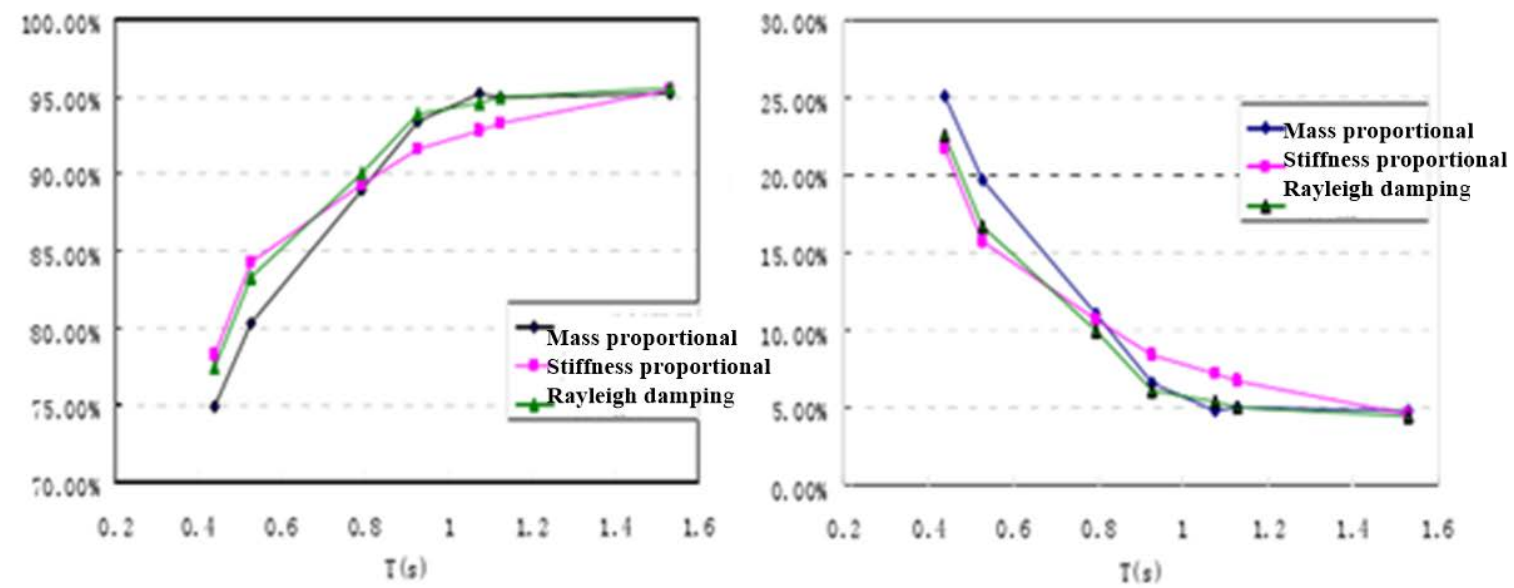

Fig. 2.The proportion of beam and column energy dissipation

\section{Conclusions}

As one of the most important parameters in the structural dynamic analysis, damping is an indicator of the energy dissipation during structural vibration. Based on the damping model that is commonly used in the finite element software, the appropriate value of the damping coefficients was discussed in the paper. Several damping models are used to solve the seismic response motion equation of a frame structure. It is shown that instantaneous stiffness damping model can bring about the maximum inter-story displacement and maximum top floor displacement, and it also produces minimum damping energy and maximum hysteresis energy. The total input energy has no significant difference between the damping models except that the modal damping model gives much less total input energy. It is demonstrated that different damping models have different seismic response results, so more precise experimental studies are necessary to find out the most reliable model. The instantaneous stiffness model is relative1y reliable in estimating structural seismic response.

\section{References}

[1] Zhu Jing qing, Zhu $\mathrm{M}$ in Calculation of complex damping response spectra from earthquake records [J]. Earthquake Engineering and Engineering Vibration, 2002 (2): 19--23.

[2] Li Penh W and Yuan feng. Analysis of seism is response of RC frame with viscous damping or complex damping [J]. Earthquake Engineering and Engineering Vibration 2007, 27(3), 54-57.

[3] ZHANG Huirlong WANG Yuanfeng Study on 3D steel frame structure with complex damping theory [J]. Journal of Shenyang Jianzhu University 2009, 25(1): 50-55.

[4] Lou Menlin, Zhang Jing. Discussion on Damping Models for Seismic Response Analysis of Long-Span Bridge [J].Journal of Vibration and Shock, 2009, (5):22-26.

[5] Arun M Puthanpurayil, Rajesh P Dhakal, Athol J Carr. Modeling of In-Structure Damping: A Review of the State-of-the-art [C]. Proceedings of the Ninth Pacific Conference on Earthquake Engineering, 2011. 